Article

\title{
Palladium-Catalyzed One-Pot Approach to 3-(Diarylmethylene)oxindoles from Propiolamidoaryl Triflate
}

\author{
Dahye Lee $^{\dagger}$, Sunhwa Park ${ }^{\dagger}$, Yoseb Yu, Kye Jung Shin and Jae Hong Seo * \\ Integrated Research Institute of Pharmaceutical Sciences, College of Pharmacy, The Catholic \\ University of Korea, Bucheon-si, Gyeonggi-do 420-743, Korea; E-Mails: dahye8821@naver.com (D.L.); \\ sunalovegs@catholic.ac.kr (S.P.); fbdytpq@catholic.ac.kr (Y.Y.); kyejung@catholic.ac.kr (K.J.S.)
}

$\dagger$ These authors contributed equally to this work.

* Author to whom correspondence should be addressed; E-Mail: jaehongseo@catholic.ac.kr;

Tel.: +82-2-2164-6531; Fax: +82-2-2164-4059.

Academic Editor: Roman Dembinski

Received: 7 July 2015 / Accepted: 31 July 2015 / Published: 3 August 2015

\begin{abstract}
Diarylmethylene)oxindoles have been synthesized from propiolamidoaryl triflate utilizing a palladium-catalyzed one-pot reaction consisting of three successive reactions: Sonogashira, Heck, and Suzuki-Miyaura. This method allows for the production of a complex skeleton of 3-(diarylmethylene)oxindole from propiolamidoaryl triflate using a commercially available aryl iodide and arylboronic acid in a simple and efficient way with moderate yield and stereoselectivity.
\end{abstract}

Keywords: 3-(Diarylmethylene)oxindole; one-pot reaction; aryl triflate; palladium-catalyzed reaction

\section{Introduction}

The oxindole scaffold, one of the major subfamilies of indoles, has been found in various biologically active natural products, therapeutics, and synthetic intermediates, which have attracted much interest from synthetic chemists to develop numerous methods for the synthesis of the unique scaffold [1-3]. Although synthetic studies on 3-(diarylmethylene)oxindoles are relatively rare, few biological activities have been reported (Figure 1). For example, TAS-301 (1) exhibits inhibitory activity of neointimal thickening following single balloon injury [4]. Recently, novel activities of the 3-(diarylmethylene)oxindoles have 
been revealed, such as AMPK activation of 2 [5] and anti-breast-cancer activity of $\mathbf{3}$ [6]. Due to these results, there is an increasing interest in the molecule's structure. Over the last decade, several groups have reported efficient synthetic methods for 3-(diarylmethylene)oxindoles utilizing a metal-catalyzed tandem reaction [7-14]. However, more efficient synthetic methods for 3-(diarylmethylene)oxindoles are required.
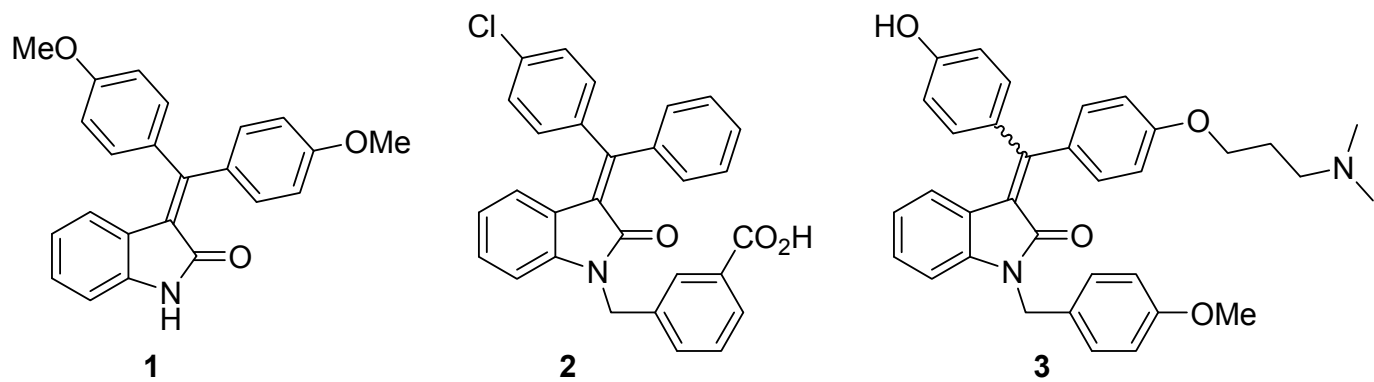

Figure 1. Biologically active 3-(diarylmethylene)oxindoles.

Due to our ongoing efforts to develop a novel domino reaction for the synthesis of biologically active heterocycles, we recently reported a palladium-catalyzed one-pot approach to 3-(diarylmethylene) oxindoles combining the Sonogashira, Heck, and Suzuki-Miyaura reactions (Scheme 1) [15]. In the onepot reaction, a Sonogashira reaction of propiolamide $4(\mathrm{X}=\mathrm{Br})$ afforded the internal alkyne 5, which was transformed into 3-(diarylmethylene)oxindole 7 by the addition of the corresponding arylboronic acid and elevation in reaction temperature. Unfortunately, the stereoselectivity for the newly formed olefin was low, yielding a mixture of $E$ - and $Z$ - isomers (7a and $7 \mathbf{b})$. This unexpected result could be rationalized by the formation of the zwitter ionic metal carbene intermediate $\mathbf{8}$, with a rotatable $\sigma$-bond at the olefin position to facilitate interconversion between two vinylpalladium species, $(Z)-6$ and (E)-6 [16-18]. This assumption led us to add a silver (I) salt during the second step of the reaction to create 5 using the Heck reaction to proceed through the cationic pathway, which is likely to reduce the formation of the carbene intermediate $\mathbf{8}$ due to the pre-existence of the cationic charge of the vinyl palladium species 6. To our delight, the addition of silver (I) salt dramatically increased stereoselectivity, strongly supporting the proposed carbene-intermediated $E / Z$ isomerization mechanism [15].

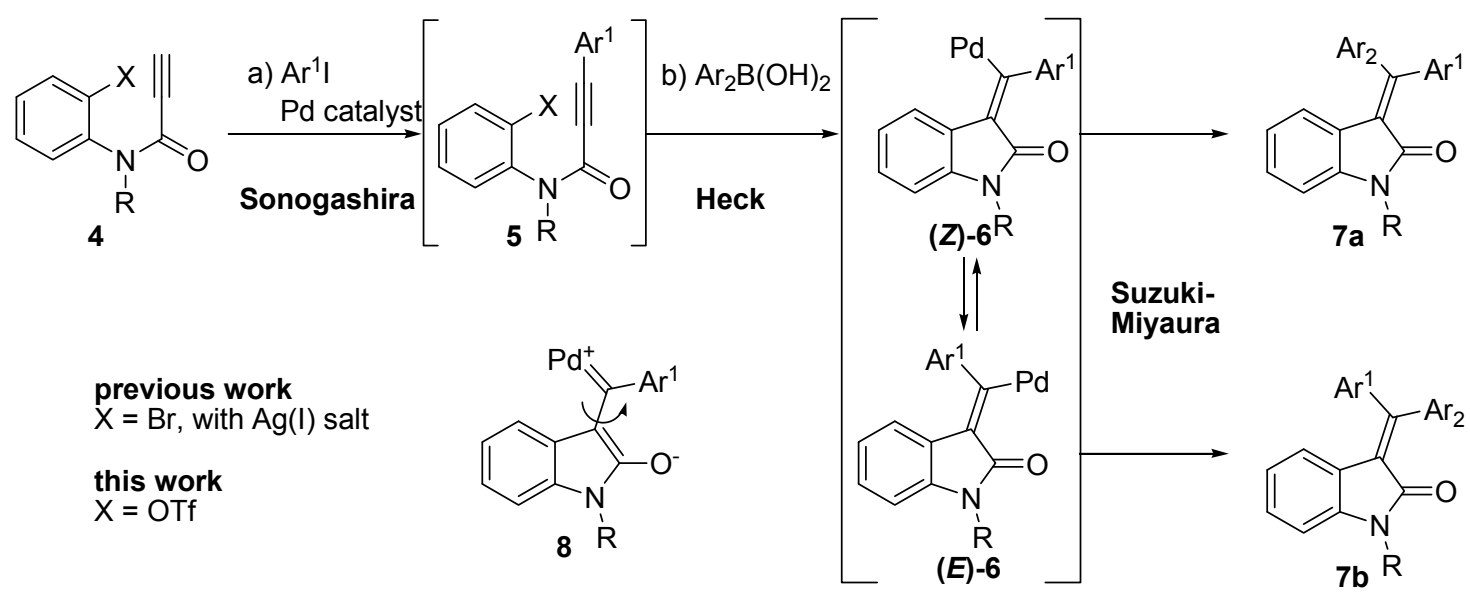

Scheme 1. Palladium-catalyzed one-pot approach to 3-(diarylmethylene)oxindoles. 
The Heck reaction of the aryl triflate has been known to follow the cationic pathway due to complete dissociation of the triflate anion from the palladium center [19]. This fact prompted us to investigate the one-pot reaction with propiolamidoaryl triflate $(4, \mathrm{X}=\mathrm{OTf})$ as a substrate, with the expectation of identifying a solution to solve the low stereoselectivity of the arylbromo substrate $(4, X=\mathrm{Br})$. Here, we describe optimization efforts for the one-pot reaction with aryl triflate $\mathbf{4}$ and substrate scope.

\section{Results and Discussion}

The aryl triflate substrate 11 was prepared from a known benzoxazolone, 9 [20], using a modification of the Overman process (Scheme 2) [21]. The addition of TBS-acetylide to 9 gave the corresponding phenoxide, which was trapped by the triflating reagent to afford TBS-propiolamide $\mathbf{1 0}$ with a yield of $88 \%$. Deprotection of the TBS group of $\mathbf{1 0}$ by TBAF under AcOH-buffered conditions gave rise to aryl triflate 11 with a yield of $83 \%$.

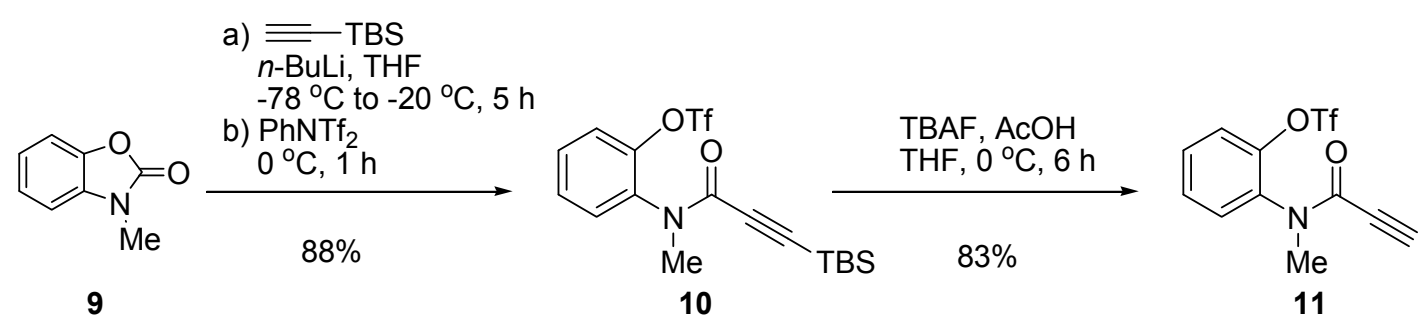

Scheme 2. Synthesis of propiolamidophenyl triflate 11.

When aryl triflate $\mathbf{1 1}$ was exposed to the previously optimized palladium-catalyzed one-pot conditions for the bromo-substrate [15], the desired 3-(diarylmethylene)oxindole $\mathbf{1 2}$ was formed with a yield of 64\%, with 1,4-benzoxazine 13 as a by-product (7\% yield) (entry 1) (Table 1). The unexpected formation of 13 was due to the base-sensitive and thermally-labile characteristics of triflates [22], causing decomposition of the triflate group of the Sonogashira adduct to the corresponding phenol compound, followed by 6-endo-dig cyclization [23,24], to give 13. To investigate the effect of the base on the reaction, a number of bases were tested. Using KOAc as a base gave a similar yield of $\mathbf{1 2}$ and $\mathbf{1 3}$ (entry 1). The weak base, $\mathrm{K}_{3} \mathrm{PO}_{4}$, increased the yield of both 12 and $\mathbf{1 3}$ to $68 \%$ and 20\%, respectively (entry 2). Interestingly, the reaction with $\mathrm{Cs}_{2} \mathrm{CO}_{3}$ was completed in a short time (2 h). However, the decomposition rate of the triflate group was much faster than the Heck/Suzuki-Miyaura domino reaction to give $\mathbf{1 3}$ as a major product with a 55\% yield but a small amount of the desired oxindole, 12 (5\% yield) (entry 4). $\mathrm{K}_{2} \mathrm{CO}_{3}$ also completed the reaction in $4 \mathrm{~h}$, but 12 was obtained as the major product with a yield of $64 \%$ (entry 5). We decided to extend the reaction time to $3 \mathrm{~h}$, expecting less decomposition of the triflate group. For $\mathrm{NaOAc}$, changing the reaction time did not increase the yield of $\mathbf{1 2}$ (61\% yield) (entry 6). In the case of $\mathrm{K}_{3} \mathrm{PO}_{4}$ as a base, the longer reaction time slightly increased the yield of $\mathbf{1 2}(72 \%)$, but substantially decreased the formation of $\mathbf{1 3}$ (5\% yield, entry $7 v$ s. 20\% yield, entry 3). The best result was obtained with $\mathrm{K}_{2} \mathrm{CO}_{3}$, which afforded $\mathbf{1 2}$ with a yield of $78 \%$ and a small amount of by-product, 13 (7\% yield) (entry 8 ). 
Table 1. Optimization of the one-pot reaction ${ }^{\mathrm{a}}$.

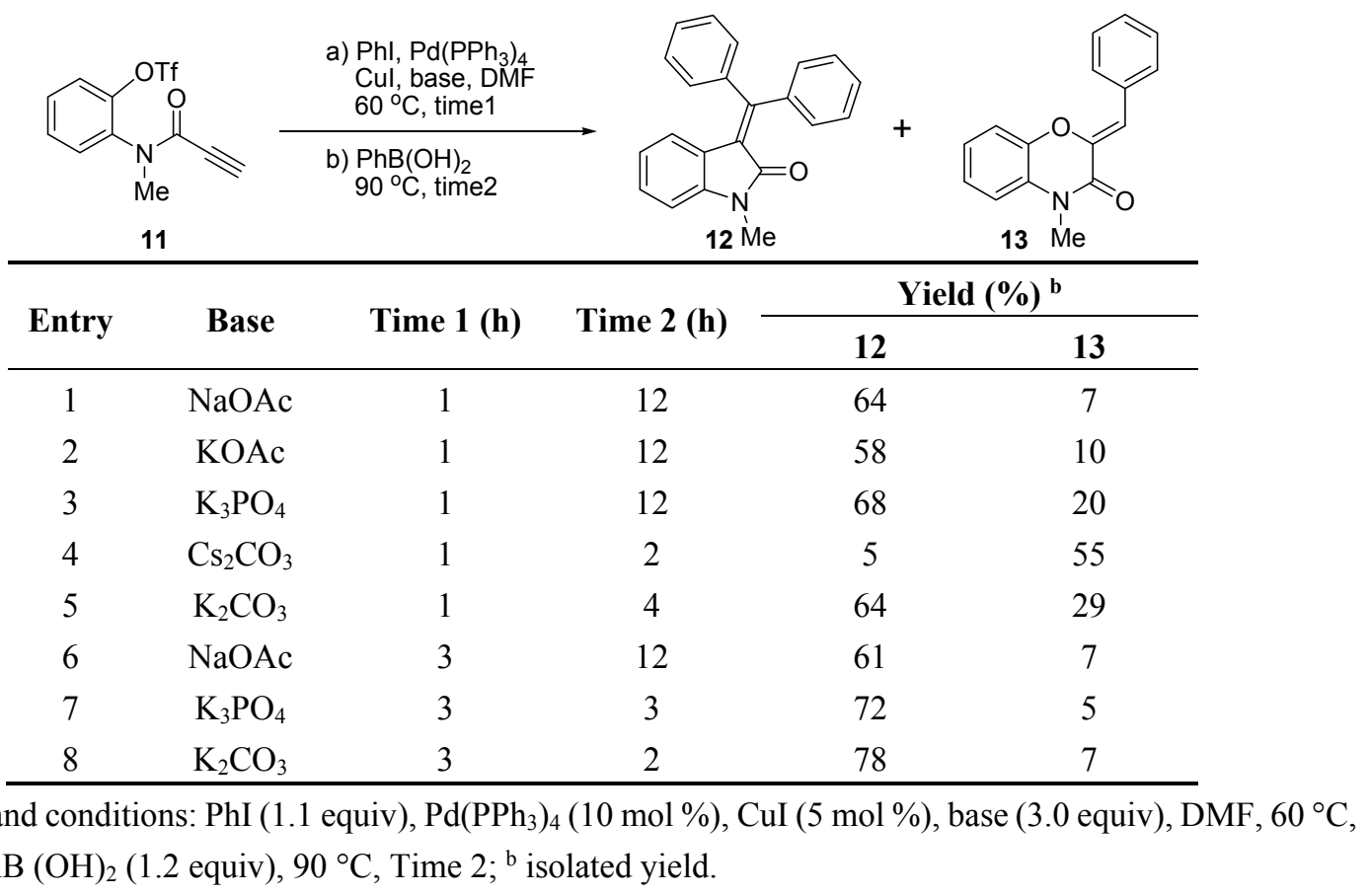

After establishing optimal conditions, we investigated the substrate scope of the one-pot reaction (Table 2). First, we applied the reaction conditions to the synthesis of symmetrically substituted 3-(diarylmethylene)oxindoles. All reactions afforded the desired oxindoles, 14a-c, with a moderate yield (42\%-55\% yields), regardless of substituents on aryliodide and arylboronic acid (entries 1-3). Comparing these results to that of the bromo substrate, $\mathbf{4}(\mathrm{X}=\mathrm{Br})$ [15], product yields from triflate 11 were slightly lower, likely due to the formation of 1,4-benzoxazine ( $<10 \%$ yield). When phenyliodide was used as a Sonogashira coupling partner, the electronic character of the substituents on arylboronic acids affected the stereochemical outcome of the products (entries 4-6). Reaction with 4-methoxyphenylboronic acid afforded 14d as a 1:1 mixture of $E / Z$ isomers (entry 4). However, electron-withdrawing chloro- and nitro-substituents on the arylboronic acids increased $E / Z$ stereoselectivity to $5: 1$ and 3:1, respectively (entries 5 and 6 ). Since the three above-mentioned reactions went through the same vinylpalladium intermediate, different stereochemical outcomes of these reactions suggested that formation of the $E / Z$ mixture of the product was not only caused by $E / Z$ isomerization of vinylpalladium species, $\mathbf{6}$. One possible explanation for this result may be the isomerization between the two products under the reaction conditions. However, when pure each isomer was re-exposed to the reaction conditions $\left(\operatorname{Pd}\left(\mathrm{PPh}_{3}\right)_{4}\right.$ (10 mol \%), $\mathrm{CuI}(5 \mathrm{~mol} \%), \mathrm{K}_{2} \mathrm{CO}_{3}$ (3.0 equiv), DMF, $90{ }^{\circ} \mathrm{C}, 2 \mathrm{~h}$ ) the interconversion between two $E / Z$ isomeric products was less than $20 \%$. This result ruled out isomerization of the product from the main contributor of low stereoselectivity [15]. However, since the reactions of the bromo substrate with the same combination of phenyliodide and arylboronic acids showed no selectivity on the $E / Z$ isomers [15], enhanced stereoselectivity by the triflate substrate strongly supports that isomerization could be reduced under the cationic pathway. Substituents of aryliodides also exerted an effect on the stereoselectivity of the products. The reaction with 4-mehoxyphenyl iodide resulted in a low $E / Z$ ratio (1.5:1) (entry 7). 4-Chloro- and 4-nitro-phenyl iodide both showed good stereoselectivity ( $E / Z=1: 5$ and 1:12, respectively) (entries 8 and 9). The stereoselectivity of the last two results are higher than those of the bromo substrate, 
which proved the usefulness of the triflate substrate for stereoselective synthesis of unsymmetrically substituted 3-(diarylmethylene)oxindole.

Table 2. Substrate scope of the one-pot reaction ${ }^{\text {a }}$.

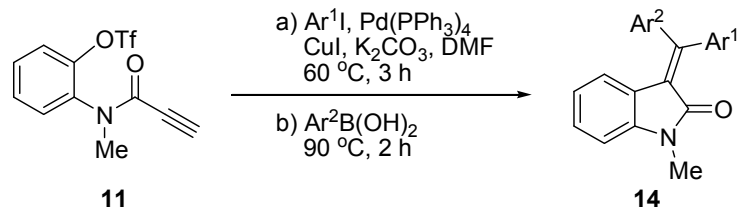

\begin{tabular}{|c|c|c|c|c|c|c|c|}
\hline \multirow{2}{*}{ Entry } & \multirow{2}{*}{$A r^{1}$} & \multirow{2}{*}{$A r^{2}$} & \multirow{2}{*}{14} & \multirow{2}{*}{ Yield (\%) } & \multirow{2}{*}{$E / Z$ Ratio $^{c}$} & \multicolumn{2}{|c|}{ Bromo Substrate (Ref. [15]) } \\
\hline & & & & & & Yield $(\%)^{b}$ & E/Z Ratio ${ }^{\mathrm{c}}$ \\
\hline 1 & 4-MeOPh & 4-MeOPh & $14 a$ & 55 & - & 85 & - \\
\hline 2 & 4-ClPh & 4- $\mathrm{ClPh}$ & $14 \mathrm{~b}$ & 54 & - & 80 & - \\
\hline 3 & $4-\mathrm{NO}_{2} \mathrm{Ph}$ & $4-\mathrm{NO}_{2} \mathrm{Ph}$ & $14 c$ & 42 & - & $67^{\mathrm{d}}$ & - \\
\hline 4 & $\mathrm{Ph}$ & 4-MeOPh & 14d & 58 & $1: 1$ & 52 & $1: 1$ \\
\hline 5 & $\mathrm{Ph}$ & 4-ClPh & $14 \mathrm{e}$ & 65 & $5: 1$ & 57 & $1: 1$ \\
\hline 6 & $\mathrm{Ph}$ & $4-\mathrm{NO}_{2} \mathrm{Ph}$ & $14 f$ & 59 & $3: 1$ & 52 & $1: 1$ \\
\hline 7 & 4-MeOPh & $\mathrm{Ph}$ & $14 d$ & 62 & $1.5: 1$ & 70 & $1.6: 1$ \\
\hline 8 & $4-\mathrm{ClPh}$ & $\mathrm{Ph}$ & $14 \mathrm{e}$ & 66 & $1: 5$ & 77 & $1: 3.4$ \\
\hline 9 & $4-\mathrm{NO}_{2} \mathrm{Ph}$ & $\mathrm{Ph}$ & $14 f$ & $62^{\mathrm{d}}$ & $1: 12$ & $73^{d}$ & $1: 10$ \\
\hline
\end{tabular}

a reagents and conditions: $\mathrm{PhI}$ (1.1 equiv), $\mathrm{Pd}\left(\mathrm{PPh}_{3}\right)_{4}(10 \mathrm{~mol} \%), \mathrm{CuI}(5 \mathrm{~mol} \%), \mathrm{K}_{2} \mathrm{CO}_{3}(3.0$ equiv), DMF, $60{ }^{\circ} \mathrm{C}, 3 \mathrm{~h} ; \mathrm{PhB}(\mathrm{OH})_{2}\left(1.2\right.$ equiv), $90{ }^{\circ} \mathrm{C}, 2 \mathrm{~h} ;{ }^{\text {b }}$ isolated yield; c ratio between isolated $E / Z$ isomers;

${ }^{\mathrm{d}} \mathrm{Heck} /$ Suzuki-Miyaura reaction was conducted at $110^{\circ} \mathrm{C}$.

\section{Experimental Section}

\subsection{General Information}

All reactions were performed under an argon atmosphere with dry solvents, unless otherwise stated. Dry diethyl ether $\left(\mathrm{Et}_{2} \mathrm{O}\right)$, tetrahydrofuran (THF), acetonitrile $(\mathrm{MeCN})$, toluene, and methylene chloride $\left(\mathrm{CH}_{2} \mathrm{Cl}_{2}\right)$ were obtained from Ultimate Solvent Purification System (JC Meyer Solvent System, Laguna Beach, CA, USA). Other dry solvents were purchased as anhydrous grade. All commercially available reagents were purchased and used without further purification. Reactions were monitored by thin-layer chromatography (TLC) on silica gel plates (Merck TLC Silica Gel 60 F254) using UV light, PMA (an ethanolic solution of phosphomolybdic acid) or ANIS (an ethanolic solution of para-anisaldehyde) as visualizing agent. Purification of products was conducted by column chromatography through silica gel 60 (0.060-0.200 mm). Melting points of all solid compounds were determined by Buchi M-565 (BÜCHI Labortechnik AG, Flawil, Switzerland). NMR spectra were obtained on Bruker AVANCE III $500 \mathrm{MHz}$ (Bruker Corporation, Billerica, MA, USA) using residual undeuterated solvent or TMS (tetramethylsilane) as an internal reference. IR spectra were recorded on a Jasco P-2000 FT-IR spectrometer (JASCO, Easton, MD, USA). High-resolution mass spectra (HR-MS) were recorded on an Agilent 6530 Q-TOF mass spectrometer (Agilent Tchnology, Santa Clara, CA, USA) using ESI (electrospray ionization) or a JEOL JMS-700 (JEOL, Tokyo, Japan) using EI (electron impact). Copies of NMR spectra of all compounds are available in the Supplementary Materials (Supplementary Figures S1-S17). 


\subsection{Preparation of Propiolamidoaryl Triflate $\mathbf{1 1}$}

2-(3-(Tert-butyldimethylsilyl)-N-methylpropiolamido)phenyl trifluoromethanesulfonate (10): To a stirred solution of TBS-acetylene $(1.30 \mathrm{~mL}, 6.96 \mathrm{mmol})$ in THF $(30 \mathrm{~mL})$ was added $n$-BuLi $(2.0 \mathrm{M}$ in hexanes, $3.2 \mathrm{~mL}, 6.4 \mathrm{mmol}$ ) at $-78{ }^{\circ} \mathrm{C}$. After $1 \mathrm{~h}$, a solution of $N$-methyl-2-benzoxzolinone (9) [20] ( $881 \mathrm{mg}, 5.91 \mathrm{mmol})$ in THF $(15 \mathrm{~mL})$ was added dropwise to the reaction mixture. Then, the reaction temperature was gradually raised to $-20{ }^{\circ} \mathrm{C}$. After $4 \mathrm{~h}$ stirring at $-20^{\circ} \mathrm{C}$, to the mixture was added a solution of $\mathrm{N}$-phenylbis(trifluoromethansulfonimide) (3.2 g, $9.0 \mathrm{mmol})$ in THF (15 mL). The reaction mixture was warmed to $0{ }^{\circ} \mathrm{C}$ and stirred for $1 \mathrm{~h}$. Then, the mixture was diluted with sat. aq. $\mathrm{NH}_{4} \mathrm{Cl}(30 \mathrm{~mL})$ and EtOAc $(300 \mathrm{~mL})$. The organic layer was separated, dried $\left(\mathrm{Na}_{2} \mathrm{SO}_{4}\right)$, filtered and concentrated under reduced pressure. The crude residue was purified by column chromatography (silica gel, hexanes:EtOAc 4:1) to afford TBS-propiolamide $\mathbf{1 0}\left(2.20 \mathrm{~g}, 88 \%\right.$ yield) as a pale yellow oil. 10: $R_{\mathrm{f}}=0.16$ (silicagel, hexanes:EtOAc 4:1); IR (film) 3726, 2984, 1652, 1496, 1423, 1361, 1308, 1212, 1140, 940, 882, 827, $778 \mathrm{~cm}^{-1} ;{ }_{1} \mathrm{H}-\mathrm{NMR}\left(500 \mathrm{MHz}, \mathrm{CDCl}_{3}, 3: 1\right.$ atropisomeric mixture): $\delta=7.47-7.31$ (m, 4H, major and minor), $3.56(\mathrm{~s}, 3 \mathrm{H}$, minor), $3.28(\mathrm{~s}, 3 \mathrm{H}$, major $), 0.99(\mathrm{~s}, 9 \mathrm{H}$, minor $), 0.68(\mathrm{~s}, 9 \mathrm{H}$, major $), 0.20$ (s, 6H, minor), -0.08 (d, $J=3.5 \mathrm{~Hz}, 6 \mathrm{H}$, major) ppm; ${ }^{13} \mathrm{C}-\mathrm{NMR}\left(125 \mathrm{MHz}, \mathrm{CDCl}_{3}\right.$, major peaks) $\delta=153.7,145.5,136.0$, 131.0, 130.1, 129.3, 122.8, 118.6 (q, $\left.{ }^{1} J_{\mathrm{C}-\mathrm{F}}=320.1 \mathrm{~Hz}\right), 97.3,96.5,36.0,25.8,16.3,-5.4 \mathrm{ppm}$; HRMS (FAB): calcd for $\mathrm{C}_{17} \mathrm{H}_{23} \mathrm{~F}_{3} \mathrm{NO}_{4} \mathrm{SSi}\left[\mathrm{M}+\mathrm{H}^{+}\right.$: 422.1069, found 422.1073 .

2-(N-methylpropiolamido)phenyl trifluoromethanesulfonate (11): To a stirred solution of TBS-propiolamide 10 (912 mg, $3.15 \mathrm{mmol})$ in THF (30 mL) was added TBAF (1.0 M in THF, $4.15 \mathrm{~mL}$, $4.15 \mathrm{mmol})$ and $\mathrm{AcOH}(0.18 \mathrm{~mL}, 3.14 \mathrm{mmol})$ at $0{ }^{\circ} \mathrm{C}$. After $24 \mathrm{~h}$, the reaction mixture was diluted with sat. aq. $\mathrm{NH}_{4} \mathrm{Cl}(30 \mathrm{~mL})$ and EtOAc $(100 \mathrm{~mL})$. The organic layer was separated, dried $\left(\mathrm{Na}_{2} \mathrm{SO}_{4}\right)$, filtered and concentrated under reduced pressure. The crude residue was purified by column chromatography (silica gel, hexanes:EtOAc 4:1) to afford propiolamide 11 (457 mg, 83\% yield) as white solid. 11: $\mathrm{mp}=70.1{ }^{\circ} \mathrm{C}$ (uncorrect); $R_{\mathrm{f}}=0.38$ (silicagel, hexanes:EtOAc 2:1); IR (film) 3727, 3229, 2923, 2852, 2109, 1778, 1652, 1494, 1421, 1368, 1309, 1213, 1138, 1092, 880, 769, 737, $681 \mathrm{~cm}^{-1} ;{ }^{1} \mathrm{H}-\mathrm{NMR}$ (500 MHz, $\mathrm{CDCl}_{3}, 3: 1$ atropisomeric mixture): $\delta=7.52-7.41(\mathrm{~m}, 4 \mathrm{H}$, major, 2H, minor), 7.38-7.36 (m, $2 \mathrm{H}$, minor), 3.59 (s, 3H, minor), 3.31 (s, 3H, major), 3.27 (s, $1 \mathrm{H}$, minor), 2.79 (s, $1 \mathrm{H}$, major) ppm; ${ }^{13} \mathrm{C}-\mathrm{NMR}$ (125 MHz, $\mathrm{CDCl}_{3}$, major peaks): $\delta=153.1,145.4,135.5,130.8,130.5,129.4,122.8,118.6\left(\mathrm{q},{ }^{1} J_{\mathrm{C}-\mathrm{F}}=\right.$ 318.6 Hz), 79.4, 75.6, 36.1 ppm; HRMS (EI): Calcd for $\mathrm{C}_{11} \mathrm{H}_{8} \mathrm{~F}_{3} \mathrm{NO}_{4} \mathrm{~S}[\mathrm{M}]^{+}$: 307.0126, found 307.0121.

\subsection{General Procedure for Palladium-Catalyzed One-Pot Reaction}

To a stirred solution of propiolamide $11(0.5 \mathrm{mmol}, 1.0$ equiv) in DMF $(5 \mathrm{~mL})$ were added the corresponding aryl iodide $\left(0.55 \mathrm{mmol}, 1.1\right.$ equiv), $\mathrm{CuI}(0.025 \mathrm{mmol}, 5 \mathrm{~mol} \%), \mathrm{K}_{2} \mathrm{CO}_{3}(1.5 \mathrm{mmol}$, 3.0 equiv) and $\mathrm{Pd}\left(\mathrm{PPh}_{3}\right)_{4}(0.05 \mathrm{mmol}, 10 \mathrm{~mol} \%)$ at $25{ }^{\circ} \mathrm{C}$. The reaction mixture was stirred at $60{ }^{\circ} \mathrm{C}$ for $3 \mathrm{~h}$. Then, the corresponding aryl boronic acid $(0.6 \mathrm{mmol}, 1.2$ equiv) was quickly added to the mixture. Reaction temperature was raised to $90^{\circ} \mathrm{C}$ or $110^{\circ} \mathrm{C}$. After $2 \mathrm{~h}$ stirring at the indicated temperature, the mixture was cooled to $25^{\circ} \mathrm{C}$ and diluted with EtOAc $(200 \mathrm{~mL})$. The organic layer was washed with $\mathrm{H}_{2} \mathrm{O}$ $(30 \mathrm{~mL} \times 3)$ and brine $(30 \mathrm{~mL})$, then dried $\left(\mathrm{Na}_{2} \mathrm{SO}_{4}\right)$, filtered and concentrated under reduced pressure. The crude residue was purified by column chromatography (silica gel, hexane:EtOAc) to yield 3(diarylmethylene)oxindoles (12 and 14a-f) and 1,4-benzoxazine 13. 
3-(Diphenylmethylene)-1-methylindolin-2-one (12): Yellow solid, $\mathrm{mp}=165.9{ }^{\circ} \mathrm{C}$ (lit. [13], 153.2-154.9 ${ }^{\circ} \mathrm{C}$ ); $R_{\mathrm{f}}=0.33$ (silica gel, hexanes:EtOAc 4:1); IR (film) 3054, 2923, 1700, 1606, $1469 \mathrm{~cm}^{-1}$; ${ }^{1} \mathrm{H}-\mathrm{NMR}\left(500 \mathrm{MHz}, \mathrm{CDCl}_{3}\right): \delta=7.45-7.41(\mathrm{~m}, 3 \mathrm{H}), 7.37-7.32(\mathrm{~m}, 7 \mathrm{H}), 7.17(\mathrm{td}, J=7.5,1.0 \mathrm{~Hz}, 1 \mathrm{H})$, $6.76(\mathrm{~d}, J=8.0 \mathrm{~Hz}, 1 \mathrm{H}), 6.68(\mathrm{td}, J=7.5,1.0 \mathrm{~Hz}, 1 \mathrm{H}), 6.42(\mathrm{dd}, J=7.5,1.0 \mathrm{~Hz}, 1 \mathrm{H}), 3.21(\mathrm{~s}, 3 \mathrm{H}) \mathrm{ppm}$; ${ }^{13} \mathrm{C}-\mathrm{NMR}\left(125 \mathrm{MHz}, \mathrm{CDCl}_{3}\right): \delta=167.0,154.7,143.5,141.5,140.1,130.1,129.5,129.3,129.2,129.1$, 128.9, 128.0, 124.4, 123.4, 123.3, 121.5, 107.8, 26.0 ppm; HRMS (ESI-TOF): calcd for $\mathrm{C}_{22} \mathrm{H}_{17} \mathrm{NO}$ [M $+\mathrm{H}]^{+}: 312.1388$, found 312.1394 .

(Z)-2-Benzylidene-4-methyl-2H-benzo[b][1,4]oxazin-3(4H)-one (13): White solid; $\mathrm{mp}=155.1{ }^{\circ} \mathrm{C}$ (lit. [25], 155-156 ${ }^{\circ} \mathrm{C}$ ); $R_{\mathrm{f}}=0.60$ (silicagel, $\mathrm{CH}_{2} \mathrm{Cl}_{2}$ ); IR (film) 3727, 2923, 2853, 1668, 1630, 1504, 1422, 1381, 1288, 1167, 1127, 1039, 743, $686 \mathrm{~cm}^{-1} ;{ }^{1} \mathrm{H}-\mathrm{NMR}\left(500 \mathrm{MHz}, \mathrm{CDCl}_{3}\right): \delta=7.85(\mathrm{~d}, J=7.5 \mathrm{~Hz}$, 2H), $7.41(\mathrm{t}, J=7.5 \mathrm{~Hz}, 2 \mathrm{H}), 7.31(\mathrm{t}, J=7.3 \mathrm{~Hz}, 1 \mathrm{H}), 7.20-7.18(\mathrm{~m}, 1 \mathrm{H}), 7.10-7.07(\mathrm{~m}, 2 \mathrm{H}), 7.02-7.00(\mathrm{~m}$, 1H), $6.96(\mathrm{~s}, 1 \mathrm{H}), 3.48(\mathrm{~s}, 3 \mathrm{H}) \mathrm{ppm} ;{ }^{13} \mathrm{C}-\mathrm{NMR}\left(125 \mathrm{MHz}, \mathrm{CDCl}_{3}\right): \delta=157.4,142.0,141.2,133.9,130.1$, 128.7, 128.3, 127.6, 123.9, 123.4, 116.1, 114.5, 113.0, 28.8 ppm; HRMS (EI): calcd for $\mathrm{C}_{16} \mathrm{H}_{13} \mathrm{NO}_{2}[\mathrm{M}]^{+}$: 251.0946 , found 251.0947 .

3-(bis(4-Methoxyphenyl)methylene)-1-methylindolin-2-one (14a): Brown solid; $\mathrm{mp}=192.2{ }^{\circ} \mathrm{C} ; R_{\mathrm{f}}=0.2$ (silica gel, hexanes:EtOAc 4:1); IR (film) 3017, 1691, 1603, $1251 \mathrm{~cm}^{-1} ;{ }^{1} \mathrm{H}-\mathrm{NMR}\left(500 \mathrm{MHz}, \mathrm{CDCl}_{3}\right)$ : $\delta=7.29-7.24(\mathrm{~m}, 4 \mathrm{H}), 7.14(\mathrm{td}, J=8.0,1.5 \mathrm{~Hz}, 1 \mathrm{H}), 6.93(\mathrm{~d}, J=9.0 \mathrm{~Hz}, 2 \mathrm{H}), 6.88(\mathrm{~d}, J=9.0 \mathrm{~Hz}, 2 \mathrm{H})$, $6.77(\mathrm{~d}, J=8.0 \mathrm{~Hz}, 1 \mathrm{H}), 6.70(\mathrm{td}, J=8.0,1.5 \mathrm{~Hz}, 1 \mathrm{H}), 6.57(\mathrm{dd}, J=7.5,0.5 \mathrm{~Hz}, 1 \mathrm{H}), 3.88$ $(\mathrm{s}, 3 \mathrm{H}), 3.84(\mathrm{~s}, 3 \mathrm{H}), 3.22(\mathrm{~s}, 3 \mathrm{H}) \mathrm{ppm} ;{ }^{13} \mathrm{C}-\mathrm{NMR}\left(125 \mathrm{MHz}, \mathrm{CDCl}_{3}\right): \delta=167.3,161.0,160.8,155.1$, $142.8,133.9,132.9,132.4,132.1,128.0,124.3,122.7,122.5,121.3,114.2,113.2,107.7,55.5,55.4,26.0$ ppm; HRMS (ESI-TOF): calcd for $\mathrm{C}_{24} \mathrm{H}_{21} \mathrm{NO}_{3}[\mathrm{M}+\mathrm{H}]^{+}: 372.1600$, found 372.1611 .

3-(bis(4-Chlorophenyl)methylene)-1-methylindolin-2-one (14b): Yellow solid; $\mathrm{mp}=134.4{ }^{\circ} \mathrm{C} ; R_{\mathrm{f}}=0.33$ (silica gel, hexanes:EtOAc 4:1); IR (film) 3054, 2927, 1699, 1606, 1486, $1089 \mathrm{~cm}^{-1}$; ${ }^{1} \mathrm{H}-\mathrm{NMR}$ $\left(500 \mathrm{MHz}, \mathrm{CDCl}_{3}\right): \delta=7.41(\mathrm{dd}, J=7.0,2.0 \mathrm{~Hz}, 2 \mathrm{H}), 7.33(\mathrm{dd}, J=7.0,2.0 \mathrm{~Hz}, 2 \mathrm{H}), 7.26-7.20(\mathrm{~m}, 5 \mathrm{H})$, $6.78(\mathrm{~d}, J=8.0 \mathrm{~Hz}, 1 \mathrm{H}), 6.73(\mathrm{td}, J=7.5,1.0 \mathrm{~Hz}, 1 \mathrm{H}), 6.52(\mathrm{~d}, J=7.5 \mathrm{~Hz}, 1 \mathrm{H}), 3.20$ (s, 3H) ppm; ${ }^{13} \mathrm{C}-\mathrm{NMR}\left(125 \mathrm{MHz}, \mathrm{CDCl}_{3}\right): \delta=166.7,151.4,143.5,139.3,138.0,135.7,135.6,131.7,131.1,129.5$, 129.4, 128.3, 125.1, 123.2, 122.8, 121.7, 108.1, 26.0 ppm; HRMS (ESI-TOF): calcd for $\mathrm{C}_{22} \mathrm{H}_{15} \mathrm{Cl}_{2} \mathrm{NO}$ $[\mathrm{M}+\mathrm{H}]^{+}:$380.0609, found 380.0612 .

3-(bis(4-Nitrophenyl)methylene)-1-methylindolin-2-one (14c): Brown solid; $\mathrm{mp}=244.5{ }^{\circ} \mathrm{C} ; R_{\mathrm{f}}=0.21$ (silica gel, hexanes:EtOAc 3:1); IR (film) 3073, 1703, 1601, 1517, 1486, 1344, $1098 \mathrm{~cm}^{-1}$; ${ }^{1} \mathrm{H}-\mathrm{NMR}$ $\left(500 \mathrm{MHz}, \mathrm{CDCl}_{3}\right): \delta=8.34(\mathrm{dd}, J=7.0,2.0 \mathrm{~Hz}, 2 \mathrm{H}), 8.24(\mathrm{dd}, J=7.0,2.0 \mathrm{~Hz}, 2 \mathrm{H}), 7.56(\mathrm{dd}, J=7.0$, $2.0 \mathrm{~Hz}, 2 \mathrm{H}), 7.48(\mathrm{dd}, J=7.0,2.0 \mathrm{~Hz}, 2 \mathrm{H}), 7.29(\mathrm{dd}, J=7.5,1.0 \mathrm{~Hz}, 1 \mathrm{H}), 6.83(\mathrm{~d}, J=8.0 \mathrm{~Hz}, 1 \mathrm{H}), 6.76$ $(\mathrm{td}, J=8.0,1.0 \mathrm{~Hz}, 1 \mathrm{H}), 6.45(\mathrm{~d}, J=7.5,1 \mathrm{H}), 3.20(\mathrm{~s}, 3 \mathrm{H}) \mathrm{ppm} ;{ }^{13} \mathrm{C}-\mathrm{NMR}\left(125 \mathrm{MHz}, \mathrm{CDCl}_{3}\right)$ : $\delta=166.0,148.4,148.1,147.1,146.3,145.6,144.3,130.9,130.6,130.4,127.5,124.8,123.63$, 123.61, 122.2, 121.4, 108.7, 26.1 ppm; HRMS (ESI-TOF): calcd for $\mathrm{C}_{22} \mathrm{H}_{15} \mathrm{~N}_{3} \mathrm{O}_{5}[\mathrm{M}+\mathrm{H}]^{+}: 402.1092$, found 402.1097 .

(Z)-3-((4-Methoxyphenyl)(phenyl)methylene)-1-methylindolin-2-one $(\boldsymbol{Z}-\mathbf{1 4 d})$ : Yellow solid; $\mathrm{mp}=153.0{ }^{\circ} \mathrm{C}$ (lit. [26], 163.8-164.3 ${ }^{\circ} \mathrm{C}$ ); $R_{\mathrm{f}}=0.24$ (silica gel, hexanes:EtOAc 4:1); IR (film) 2931, 1696, 1604, 
$1250 \mathrm{~cm}^{-1} ;{ }^{1} \mathrm{H}-\mathrm{NMR}\left(500 \mathrm{MHz}, \mathrm{CDCl}_{3}\right): \delta=7.46-7.40(\mathrm{~m}, 3 \mathrm{H}), 7.32-7.29(\mathrm{~m}, 4 \mathrm{H}), 7.15$ (t, $J=13.0 \mathrm{~Hz}, 1 \mathrm{H}), 6.88(\mathrm{dd}, J=7.0,2.0 \mathrm{~Hz}, 2 \mathrm{H}), 6.76(\mathrm{~d}, J=8.0 \mathrm{~Hz}, 1 \mathrm{H}), 6.66(\mathrm{t}, J=7.5 \mathrm{~Hz}, 1 \mathrm{H}), 6.32$ $(\mathrm{d}, J=7.5 \mathrm{~Hz}, 1 \mathrm{H}), 3.84(\mathrm{~s}, 3 \mathrm{H}), 3.23(\mathrm{~s}, 3 \mathrm{H}) \mathrm{ppm} ;{ }^{13} \mathrm{C}-\mathrm{NMR}\left(125 \mathrm{MHz}, \mathrm{CDCl}_{3}\right): \delta=167.1,161.0,154.9$, 143.0, 141.7, 132.7, 131.9, 129.9, 129.3, 129.0, 128.4, 123.9, 123.0, 121.4, 113.2, 107.7, 55.4, 26.0 ppm; HRMS (ESI-TOF): Calcd for $\mathrm{C}_{23} \mathrm{H}_{19} \mathrm{NO}_{2}[\mathrm{M}+\mathrm{H}]^{+}: 342.1494$, found 342.1503.

(E)-3-((4-Methoxyphenyl)(phenyl)methylene)-1-methylindolin-2-one (E-14d): Yellow solid; $\mathrm{mp}=162.7{ }^{\circ} \mathrm{C}$ (lit. [13], 163.8-164.3 ${ }^{\circ} \mathrm{C}$ ); $R_{\mathrm{f}}=0.26$ (silica gel, hexanes:EtOAc 4:1); IR (film) 3053, 2927, 1696, 1603, 1508, 1469, $1250 \mathrm{~cm}^{-1}$; ${ }^{1} \mathrm{H}-\mathrm{NMR}\left(500 \mathrm{MHz}, \mathrm{CDCl}_{3}\right): \delta=7.39-7.25(\mathrm{~m}, 7 \mathrm{H}), 7.17(\mathrm{td}, J=7.5,1.5 \mathrm{~Hz}$, $1 \mathrm{H}), 6.93(\mathrm{dd}, J=7.0,2.0 \mathrm{~Hz}, 2 \mathrm{H}), 6.78-6.67(\mathrm{~m}, 3 \mathrm{H}), 3.87(\mathrm{~s}, 3 \mathrm{H}), 3.20(\mathrm{~s}, 3 \mathrm{H}) \mathrm{ppm} ;{ }^{13} \mathrm{C}-\mathrm{NMR}$ $\left(125 \mathrm{MHz}, \mathrm{CDCl}_{3}\right): \delta=167.1,160.8,154.9,143.3,140.6,133.6,132.7,131.7,130.5,129.3,129.0,128.6$, 127.9, 122.9, 121.4, 114.3, 107.8, 55.5, 26.0 ppm; HRMS (ESI-TOF): calcd for $\mathrm{C}_{23} \mathrm{H}_{19} \mathrm{NO}_{2}$ $[\mathrm{M}+\mathrm{H}]^{+}: 342.1494$, found 342.1498 .

(Z)-3-((4-Chlorophenyl)(phenyl)methylene)-1-methylindolin-2-one $(\boldsymbol{Z}-\mathbf{1 4 e})$ : Yellow solid; $\mathrm{mp}=59.0^{\circ} \mathrm{C}$ : $R_{\mathrm{f}}=0.37$ (silica gel, hexanes:EtOAc 4:1); IR (film) 3054, 1698, 1606, 1486, 1335, $1090 \mathrm{~cm}^{-1}$; ${ }^{1} \mathrm{H}-\mathrm{NMR}\left(500 \mathrm{MHz}, \mathrm{CDCl}_{3}\right): \delta=7.48-7.43(\mathrm{~m}, 3 \mathrm{H}), 7.35-7.27(\mathrm{~m}, 6 \mathrm{H}), 7.17(\mathrm{td}, J=7.5,1.0 \mathrm{~Hz}, 1 \mathrm{H})$, $6.77(\mathrm{~d}, J=8.0 \mathrm{~Hz}, 1 \mathrm{H}), 6.68(\mathrm{td}, J=7.5,1.0 \mathrm{~Hz}, 1 \mathrm{H}), 6.42(\mathrm{dd}, J=7.5,0.5 \mathrm{~Hz}, 1 \mathrm{H}), 3.20$ (s, 3H) ppm; ${ }^{13} \mathrm{C}-\mathrm{NMR}\left(125 \mathrm{MHz}, \mathrm{CDCl}_{3}\right): \delta=166.9,153.0,143.5,141.0,138.4,135.3,131.6,129.51,129.50,129.2$, 129.1, 128.2, 124.7, 123.4, 123.2, 121.6, 107.9, 26.0 ppm; HRMS (EI): Calcd for $\mathrm{C}_{22} \mathrm{H}_{16} \mathrm{CINO}$ [M] $]^{+}$ 345.0920, found 345.0916 .

(E)-3-((4-Chlorophenyl)(phenyl)methylene)-1-methylindolin-2-one $(\boldsymbol{E}-14 \mathbf{e})$ : Yellow solid; $\mathrm{mp}=54.8^{\circ} \mathrm{C}$; $R_{\mathrm{f}}=0.35$ (silica gel, hexanes:EtOAc 4:1); ${ }^{1} \mathrm{H}-\mathrm{NMR}\left(500 \mathrm{MHz}, \mathrm{CDCl}_{3}\right): \delta=7.41-7.18(\mathrm{~m}, 9 \mathrm{H}), 7.19$ $(\mathrm{td}, J=7.5,1.0 \mathrm{~Hz}, 1 \mathrm{H}), 6.78(\mathrm{~d}, J=7.5 \mathrm{~Hz}, 1 \mathrm{H}), 6.73(\mathrm{td}, J=7.5,1.0 \mathrm{~Hz}, 1 \mathrm{H}), 6.53$ (dd, $J=7.5,0.5 \mathrm{~Hz}$, $1 \mathrm{H}), 3.20(\mathrm{~s}, 3 \mathrm{H}) \mathrm{ppm} ;{ }^{13} \mathrm{C}-\mathrm{NMR}\left(125 \mathrm{MHz}, \mathrm{CDCl}_{3}\right): \delta=166.8,153.0,143.6,139.8,139.7,135.5,131.1$, 130.2, 129.42, 129.37, 129.2, 128.1, 124.7, 123.2, 123.0, 121.6, 108.0, 26.0 ppm; HRMS (EI): Calcd for $\mathrm{C}_{22} \mathrm{H}_{16} \mathrm{ClNO}[\mathrm{M}]^{+}:$345.0920, found 345.0919.

(Z)-1-Methyl-3-((4-nitrophenyl)(phenyl)methylene)indolin-2-one $(\boldsymbol{Z}-\mathbf{1 4 f})$ : Yellow solid; $\mathrm{mp}=186.6{ }^{\circ} \mathrm{C}$; $R_{\mathrm{f}}=0.28$ (silica gel, hexanes:EtOAc 4:1); IR (film) 3057, 1699, 1603, 1510, $1342 \mathrm{~cm}^{-1}$; ${ }^{1} \mathrm{H}-\mathrm{NMR}$ $\left(500 \mathrm{MHz}, \mathrm{CDCl}_{3}\right): \delta=8.22(\mathrm{dd}, J=7.0,1.5 \mathrm{~Hz}, 2 \mathrm{H}), 7.50-7.44(\mathrm{~m}, 5 \mathrm{H}), 7.32(\mathrm{dd}, J=8.0,2.0 \mathrm{~Hz}, 2 \mathrm{H})$, $7.22(\mathrm{t}, J=7.5 \mathrm{~Hz}, 1 \mathrm{H}), 6.80(\mathrm{~d}, J=8.0 \mathrm{~Hz}, 1 \mathrm{H}), 6.73(\mathrm{t}, J=7.75 \mathrm{~Hz}, 1 \mathrm{H}), 6.52(\mathrm{~d}, J=8.0 \mathrm{~Hz}, 1 \mathrm{H}), 3.20$ (s, 3H) ppm; ${ }^{13} \mathrm{C}-\mathrm{NMR}\left(125 \mathrm{MHz}, \mathrm{CDCl}_{3}\right): \delta=166.6,150.8,147.8,147.0,143.9,140.0,130.7,129.9$, 129.8, 129.4, 129.2, 126.1, 123.7, 123.4, 122.4, 121.9, 108.2, 26.0 ppm; HRMS (ESI-TOF): Calcd for $\mathrm{C}_{22} \mathrm{H}_{16} \mathrm{~N}_{2} \mathrm{O}_{3}[\mathrm{M}+\mathrm{H}]^{+}:$357.1239, found 357.1249.

(E)-1-Methyl-3-((4-nitrophenyl)(phenyl)methylene)indolin-2-one $(\boldsymbol{E}-14 f)$ : Yellow solid; $\mathrm{mp}=201.2{ }^{\circ} \mathrm{C}$ (lit. [13], 198.8-200.8 ${ }^{\circ} \mathrm{C}$ ); $R_{\mathrm{f}}=0.25$ (silica gel, hexanes:EtOAc 4:1); IR (film) 3056, 1701, 1604, 1519, 1469, $1345 \mathrm{~cm}^{-1} ;{ }^{1} \mathrm{H}-\mathrm{NMR}\left(500 \mathrm{MHz}, \mathrm{CDCl}_{3}\right): \delta=8.30$ (t, $\left.J=4.25 \mathrm{~Hz}, 2 \mathrm{H}\right), 7.54(\mathrm{~d}, J=9.0 \mathrm{~Hz}, 2 \mathrm{H})$, $7.41-7.21(\mathrm{~m}, 6 \mathrm{H}), 6.80(\mathrm{~d}, J=8.0 \mathrm{~Hz}, 1 \mathrm{H}), 6.71(\mathrm{t}, J=7.75 \mathrm{~Hz}, 1 \mathrm{H}), 6.36(\mathrm{~d}, J=7.5 \mathrm{~Hz}, 1 \mathrm{H}), 3.21$ (s, 3H) ppm; ${ }^{13} \mathrm{C}-\mathrm{NMR}\left(125 \mathrm{MHz}, \mathrm{CDCl}_{3}\right): \delta=166.4,150.9,148.2,147.9,143.9,138.8,130.6,130.0$, 
129.9, 129.7, 128.3, 125.7, 124.4, 123.3, 122.4, 121.8, 108.3, 26.1 ppm; HRMS (EI): calcd for $\mathrm{C}_{22} \mathrm{H}_{16} \mathrm{~N}_{2} \mathrm{O}_{3}[\mathrm{M}]^{+}:$356.1161, found 356.1161 .

\section{Conclusions}

In conclusion, we developed an efficient method for the synthesis of 3-(diarylmethylene)oxindoles from propiolamidoaryl triflate. Aryl triflate showed greater $E / Z$ selectivity than those of the bromo substrate, which proved itself as another solution for low stereoselectivity of the palladium-catalyzed one-pot reaction, and supports the previously proposed isomerization mechanism of vinylpalladium species via a zwitter ionic metal carbene intermediate.

\section{Supplementary Materials}

Supplementary materials can be accessed at: http://www.mdpi.com/1420-3049/20/08/14022/s1.

\section{Acknowledgments}

This research was supported by the Basic Science Research Program through the National Research Foundation of Korea (NRF) funded by the Ministry of Science, ICT \& Future Planning (NRF-2014R1A1A1038332), and Research Fund 2011 of The Catholic University of Korea

\section{Author Contributions}

J.H.S. and K.J.S. conceived and designed the experiments; D.L. and S.P. performed experiments; Y.Y. analyzed data; J.H.S. wrote the paper.

\section{Conflicts of Interest}

The authors declare no conflict of interest.

\section{References}

1. Marti, C.; Carreira, E.M. Construction of spiro[pyrrolidine-3,3'-oxindoles]-Recent Application to the synthesis of oxindole alkaloids. Eur. J. Org. Chem. 2003, 12, 2209-2219.

2. Kein, J.E.M.; Taylor, R.J.K. Transition-metal-mediated Routes to 3,3-disubstituted oxindoles through anilide cyclization. Eur. J. Org. Chem. 2011, 34, 6821-6841.

3. Ziarani, G.M.; Gholamzadeh, P.; Lashgari, N.; Hajiabbast, P. Oxindole as starting material in organic synthesis. ARKIVOC 2013, 1, 470-535.

4. Sasaki, E.; Miyoshi, K.; Nozawa, Y.; Kanda, A.; Nakano, K.; Yamasaki, Y.; Miyake, H.; Mausuura, N. TAS-301, a new synthetic inhibitor of neointimal thickening after balloon injury, inhibits calcium-dependent signal transduction and cytoskeletal reorganization. Pharmacology 2001, 63, 17-27.

5. Yu, L.F.; Li, Y.Y.; Su, M.B.; Zhang, M.; Zhang, W.; Zhang, L.N.; Pang, T.; Zhang, R.T.; Liu, B.; Li, J.Y.; et al. Development of novel alkene oxindole derivatives as orally efficacious AMP-activated protein kinase activators. ACS Med. Chem. Lett. 2013, 4, 475-480. 
6. Pal, A.; Ganguly, A.; Ghosh, A.; Yousuf, M.; Rathore, B.; Banerjee, R.; Adhikari, S. Bis-arylidene oxindoles as anti-breast-cancer agents acting via the estrogen receptor. Chem. Med. Chem. 2014, 9 , 727-732.

7. Yanada, R.; Obika, S.; Oyama, M.; Takemoto, Y. Stereoselective synthesis of 3-alkylideneoxindoles using tandem In-mediated carbometalation and Pd-catalyzed cross-Coupling reaction. Org. Lett. 2004, 6, 2825-2828.

8. Yanada, R.; Obika, S.; Inokuma, T.; Yanada, K.; Yamashita, M.; Ohta, S.; Takemoto, Y. Stereoselective synthesis of 3-alkylideneoxindoles via palladium-catalyzed domino reactions. J. Org. Chem. 2005, 70, 6972-6975.

9. Cheung, W.S.; Patch, R.J.; Player, M.R. A tandem Heck-carbocyclization/Suzuki-coupling approach to the Stereoselective synthesis of asymmetric 3,3-(diarylmethylene)indolinones. J. Org. Chem. 2005, 70, 3741-3744.

10. Shintani, R.; Yamagami, T.; Hayashi, T. Rhodium-catalyzed multicomponent-coupling reactions involving a carborhodation-cross-coupling sequence. Org. Lett. 2006, 8, 4799-4801.

11. Pinto, A.; Neuville, L.; Zhu, J. Palladium-catalyzed three-component synthesis of 3-(diarylmethylene)oxindoles through a domino Sonogashira/carbopalladation/C-H activation/C-C bond-forming sequence. Angew. Chem. Int. Ed. 2007, 46, 3291-3295.

12. Miura, T.; Toyoshima, T.; Takahashi, Y.; Murakami, M. Stereoselective synthesis of 3-alkylideneoxindoles by palladium-catalyzed cyclization reaction of 2-(alkynyl)aryl isocyanates with organoboron reagents. Org. Lett. 2008, 10, 4887-4889.

13. Tang, S.; Peng, P.; Zhong, P.; Li, J.H. Palladium-catalyzed C-H functionalization of $\mathrm{N}$-arylpropiolamides with aryliodonium salts: Selective synthesis of 3-(1-arylmethylene)oxindoles. J. Org. Chem. 2008, 73, 5476-5480.

14. Song, R.J.; Liu, Y.; Li, R.J.; Li, J.H. Selective synthesis of 3-methyleneindolin-2-ones by one-pot multicatalytic processes. Tetrahedron Lett. 2009, 50, 3912-3916.

15. Dong, G.R.; Park, S.; Lee, D.; Shin K. J.; Seo, J.H. Synthesis of 3-(diarylmethylene)oxindoles via a palladium-catalyzed one-pot reaction: Sonogashira-Heck-Suzuki-Miyaura combined reaction. Synlett 2013, 24, 1993-1997.

16. Zargarian, D.; Alper, H. Palladium-catalyzed hydrocarboxylation of alkynes with formic acid. Organometallics 1993, 12, 712-724.

17. Cacchi, S.; Fabrizi, G.; Marinelli, F.; Moro, L.; Pace, P. Palladium-catalyzed hydroarylation and hydrovinylation of 3,3-dialkoxy-1-aryl-1-propynes. An approach to 3-aryl- and 3-vynylquinolines. Tetrahedron 1996, 52, 10225-10240.

18. Amatore, C.; Bensalem, S.; Ghalem, S.; Jutand, A. Mechanism of the carbopalladation of alkynes by aryl-palladium complexes. J. Organomet. Chem. 2004, 689, 4642-4646.

19. Dounay, A.B.; Overman, L.E. The asymmetric intramolecular Heck reaction in natural product total synthesis. Chem. Rev. 2003, 103, 2945-2963.

20. Sam, J.; Plampin, J.N.; Poos, G.I. Methylated 2-Amino-5-chlorobenzoxazoles. J. Org. Chem. 1958, 23, 1500-1503.

21. Overman, L.E.; Peterson, E.A. Enantioselective synthesis of (-)-idiospermuline. Tetrahedron 2003, 59, 6905-6919. 
22. Suzuki, A. Recent advances in the cross-coupling reactions of organoboron derivatives with organic electrophiles, 1995-1998. J. Organomet. Chem. 1999, 576, 147-168.

23. Chowdhury, C.; Chaudhuri, G.; Guha, S.; Mukherjee, A.K.; Kundu, N.G. Palladium-catalyzed heteroannulation leading to heterocyclic structures with two heteroatoms: A highly convenient and facile method for a totally region- and stereoselective synthesis of (Z)-2,3-dihydro-2-(ylidene)-1,4benzo- and -naphtho[2,3-b]dioxnis. J. Org. Chem. 1998, 63, 1863-1871.

24. Kumar, K.S.; Rambabu, D.; Prasad, B.; Mujahid, M.; Krishna, G.R.; Rao, M.V.B.; Reddy, C.M.; Vanaja, G.R.; Kalle, A.M.; Pal, M. A new approach to construct a fused 2-ylidene chromene ring: highly regioselective synthesis of novel chromeno quinoxalines. Org. Biomol. Chem. 2012, 10, 4774-4781.

25. Loudon, J.D.; Ogg, J. 2,3-Dihydro-3-oxobenz-1,4-oxaxines. J. Chem. Soc. 1955, 739-744, doi:10.1039/JR9550000739.

26. Tang, D.J.; Tang, B.X., Li, J.H. Selective synthesis of 3-aryl quinolin-2(1H)-ones and 3-(1-arylmethylene)oxindoles involving a 2-fold arene C-H activation process J. Org. Chem. 2009, $74,6749-6755$.

Sample Availability: Not available.

(C) 2015 by the authors; licensee MDPI, Basel, Switzerland. This article is an open access article distributed under the terms and conditions of the Creative Commons Attribution license (http://creativecommons.org/licenses/by/4.0/). 